\title{
Communication
}

\section{Synthesis of Aminopropyltriethoxysilyl-Substituted Imines and Amides}

\author{
Surya R. Banks, J. Tanner Morningstar and Mark E. Welker *(i)
}

check for updates

Citation: Banks, S.R.; Morningstar, J.T.; Welker, M.E. Synthesis of AminopropyltriethoxysilylSubstituted Imines and Amides. Molbank 2021, 2021, M1251. https://doi.org/10.3390/M1251

Academic Editor: Hidenori Tanaka

Received: 28 June 2021

Accepted: 13 July 2021

Published: 18 July 2021

Publisher's Note: MDPI stays neutral with regard to jurisdictional claims in published maps and institutional affiliations.

Copyright: (c) 2021 by the authors. Licensee MDPI, Basel, Switzerland. This article is an open access article distributed under the terms and conditions of the Creative Commons Attribution (CC BY) license (https:/ / creativecommons.org/licenses/by/ $4.0 /)$.
Department of Chemistry and Center for Functional Materials, Wake Forest University, Winston-Salem, NC 27101, USA; suryabanks@gmail.com (S.R.B.); mornjt20@wfu.edu (J.T.M.)

* Correspondence: welker@wfu.edu; Tel.: +1-336-702-1953

Abstract: A series of small molecules containing aminopropyltriethoxysilyl-substituted imines and amides were synthesized so that they could potentially be incorporated into self-assembled monolayers (SAMs) on metal oxide surfaces. Simple one-step imine preparations and two-step amide preparations are reported here.

Keywords: aminopropyltriethoxysilane; amide; imine; self-assembled monolayer

\section{Introduction}

A series of small molecules containing aminopropyltriethoxysilane (APTES) linkers were synthesized so that they could potentially be incorporated into self-assembled monolayers (SAMS) on metal oxide surfaces. Trialkoxysilanes are widely used to modify metal oxide surfaces since they readily react with surface hydroxyl groups to release the alkanol and provide a piano stool trialkoxysilane linkage to the surface [1-11]. Two main structural aspects of the small molecules to be synthesized were considered: (1) ease of synthesis of the small molecule, i.e., where possible, one-pot reactions from inexpensive, commercially available starting materials, and (2) presentation of a variety of aromatic functional groups that would be of interest to others working to use SAMS as components of materials for molecular electronics or sensing applications. Imines that contain both electron-donating and -withdrawing substituents on a benzene ring, as well as a number of imines with nitrogen heterocycles as the aromatic component, were prepared. Amides were prepared containing pyridine, furan, and thiophene rings as part of the aromatic component.

\section{Results and Discussion}

To satisfy the above criteria, we ended up performing two series of reactions: (1) involving treatment of aromatic aldehydes with aminopropyltriethoxysilane (APTES) in dichloromethane (DCM) in the presence of anhydrous sodium sulfate as a drying agent and (2) involving treatment of aromatic carboxylic acids with $N$-hydroxysuccinimide (NHS) and dicyclohexylcarbodiimide (DCC) followed by APTES.

\subsection{Imines Prepared from 4-Acyl Substituted Benzaldehydes}

A variety of 4-acyl substituted benzaldehydes are commercially available and we investigated the use of a number of them in this imine forming reaction (Scheme 1). 4-Formylbenzamides (2,4), -benzoates (3), and -acetophenone (5) all produced products in high yield. We also tried using terephthalaldehyde in this reaction but it yielded essentially a 1:1:1 mixture of unreacted dialdehyde, mono imine/mono aldehyde and diimine when treated with 1 equivalent of APTES. When treated with two equivalents of APTES, dialdehyde yielded the diimine (6) in good yield. 4-Formylbenzoic acid required ethanol rather than DCM as a solvent to test this reaction and did not produce any imine product presumably due to rapid acid-base chemistry that would occur between it and APTES. 




1

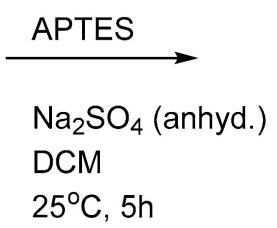

$$
\begin{aligned}
& 3 \mathrm{R}=\mathrm{OMe}(82 \%) \\
& \mathbf{4} \mathrm{R}=\operatorname{NHMe}(68 \%) \\
& 5 \mathrm{R}=\operatorname{Me}(81 \%)
\end{aligned}
$$$$
\mathrm{R}=\mathrm{NH}_{2}(87 \%)
$$



$652 \%$

Scheme 1. Preparation of aminopropyltriethoxysilyl-substituted imines from 4-acylbenzaldehydes.

\subsection{Imines Prepared from Cyano and Nitro Substituted Benzaldehydes}

Earlier we had reported that a 4-cyanophenyl aminopropyltriethoxysilyl imine could be prepared and incorporated into a molecular rectifier so we wanted to use this method prepare a number of different imines from benzaldehydes with strong electron withdrawing groups (7) (Scheme 2) [2]. As expected, these reactions proceeded well to produce imines (8-11) that can be isolated in high yield. As with all of these imines, they are best stored for long periods of time under nitrogen in a refrigerator.
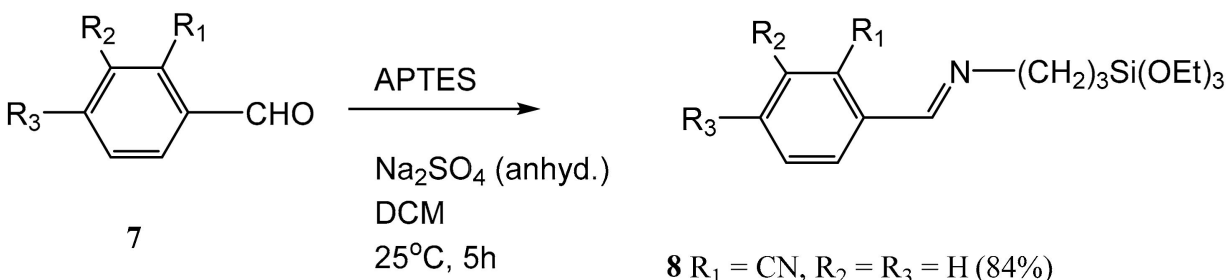

$$
\begin{aligned}
& \mathbf{8} R_{1}=C N, R_{2}=R_{3}=H(84 \%) \\
& 9 R_{2}=C N, R_{1}=R_{3}=H(80 \%) \\
& 10 R_{3}=C N, R_{2}=F, R_{1}=H(68 \%) \\
& 11 R_{3}=N O_{2}, R_{1}=R_{2}=H(86 \%)
\end{aligned}
$$

Scheme 2. Preparation of aminopropyltriethoxysilyl-substituted imines from electron withdrawing group substituted benzaldehydes.

\subsection{Imines Prepared from Heterocyclic Aromatic Aldehydes}

Imines formed from isonicotinaldehyde and pyridazine carbaldehyde as well as those prepared from fused heterocyclic aldehydes $(13,14,18$ and 19) were all isolated in slightly lower but still acceptable yields presumably due to the presence of the more electron rich aromatic rings (Scheme 3). Whereas heterocyclic substituents on benzaldehyde produced imines (15-17) in yields like we observed for reactions of benzaldehydes containing electron withdrawing substituents. 

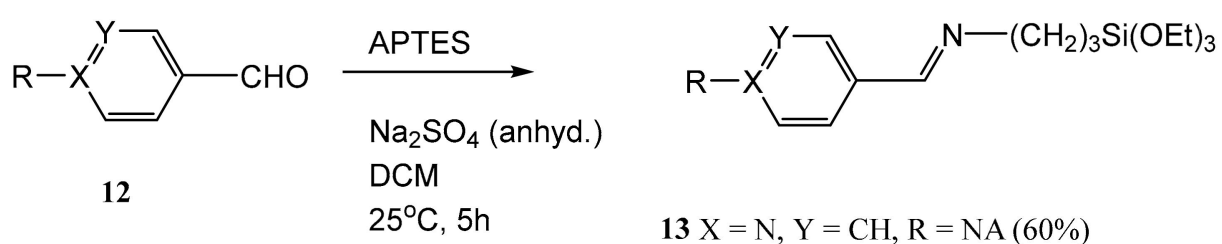

$$
\begin{aligned}
& 13 X=N, Y=C H, R=N A(60 \%) \\
& 14 X=Y=N, R=N A(65 \%) \\
& 15 X=C, Y=C H, R=4-p y r i d i n y l ~(84 \%) \\
& 16 X=C, Y=C H, R=\text { imidazolyl }(73 \%) \\
& 17 X=C, Y=C H, R=\text { pyrazolyl }(80 \%)
\end{aligned}
$$

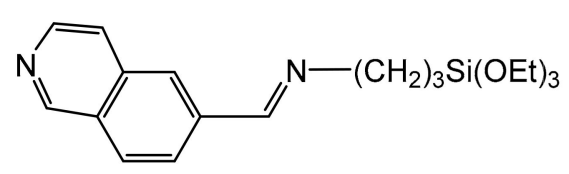

$18(60 \%)$

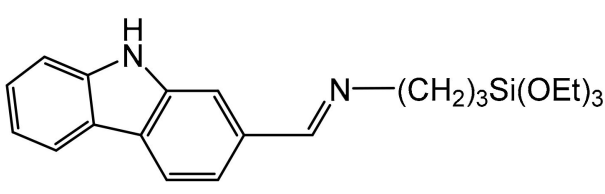

$19(65 \%)$

Scheme 3. Preparation of aminopropyltriethoxysilyl-substituted imines from heterocyclic aromatic aldehydes (NA = Not applicable).

\subsection{Imines Prepared from Disubstituted Benzaldehydes}

Trialkoxysilanes bearing substituents on the benzene ring that are conformationally restricted might prove useful for self-assembly on surfaces so we prepared a couple of imines from ortho substituted 4-formyl benzoates (Scheme 4). However, the imine prepared from methyl 2-hydroxy-4-formyl benzoate (21) showed no evidence of intramolecular hydrogen bonding (no line broadening) by NMR when evaluated from $-30{ }^{\circ} \mathrm{C}$ to $40{ }^{\circ} \mathrm{C}$ in $\mathrm{CDCl}_{3}$; therefore, the $\mathrm{CO}_{2} \mathrm{Me}$ group can presumably freely rotate around the $\mathrm{CO}_{2} \mathrm{Me}$ phenyl C-C bond.
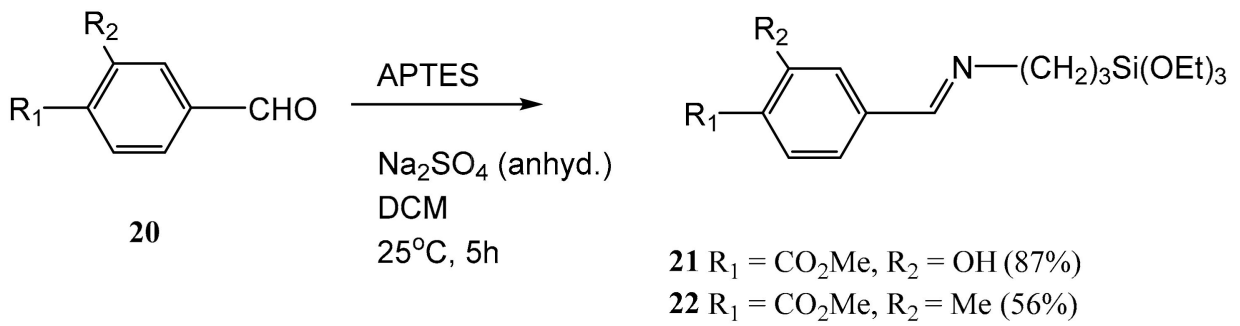

Scheme 4. Preparation of aminopropyltriethoxysilyl-substituted imines from disubstituted benzaldehydes.

\subsection{Attempts to Prepare Imines from Acetophenones Rather Than Benzaldehydes}

Lastly, for imines, we investigated the reactions of two acetophenones rather than benzaldehydes in this imine forming reaction. Neither 4-hydroxyacetophenone nor 4-carbomethoxy acetophenone produced any imine product when stirred under our standard conditions. Likewise reflux in DCM overnight with $\mathrm{MgSO}_{4}$ just produced unreacted acetophenones with traces of other compounds noted by NMR (Supplementary Materials). We did notice that 4-hydroxyacetophenone and APTES when mixed neat slowly reacted to produce an orange solid which we presumed to be the salt formed from proton transfer.

\subsection{Amides Prepared from Aromatic Carboxylic Acids and APTES}

Finally, we wanted to prepare a few aromatic amides linked to trialkoxysilanes (Scheme 5) since the imines we have prepared here might be sensitive to acid catalyzed degradation if bonded to acidic surfaces. To prepare these amides, we treated aromatic carboxylic acids with $\mathrm{N}$-hydroxysuccinimide (NHS) and dicyclohexylcarbodiimide (DCC) followed by APTES. While the isolated yields of these reactions are not as high as the 
imine forming reactions reported above yields around $50 \%$ were obtained regardless of the aromatic acid used.

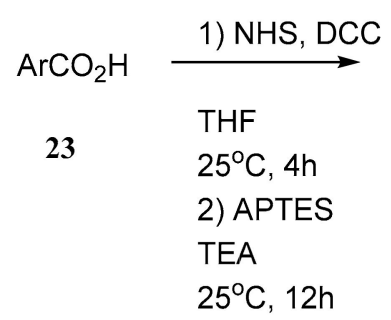<smiles>CC[SiH2]CCCNC(=O)Br</smiles>

$24 \mathrm{ArCO}_{2} \mathrm{H}=$ isonicotinic acid (51\%)

$25 \mathrm{ArCO}_{2} \mathrm{H}=$ benzofuran-5-carboxylic acid (55\%)

$26 \mathrm{ArCO}_{2} \mathrm{H}=$ benzo-[b]-thiophene-5-carboxylic acid (49\%)

Scheme 5. Preparation of aminopropyltriethoxysilyl-substituted amides.

\section{Experimental}

3.1. General Methods

NMR spectra were obtained on Bruker $300 \mathrm{MHz}$ and $400 \mathrm{MHz}$ spectrometers and mass spectrometry was performed on a Thermo LTQ Orbitrap XL. All reagents and materials were obtained from the suppliers listed below. Fischer Scientific: sodium sulfate, dichloromethane, tetrahydrofuran, $N$-hydroxysuccinimide, $N, N$-dicyclohexylcarbodiimide, triethylamine; gelest: aminopropyltriethoxysilane; Ambeed: methyl 4 acetylbenzoate, 4 acetyl benzaldehyde, methyl 4-formyl-2-methylbenzoate, 2-fluoro-4-formyl benzonitrile, and methyl 4-formyl-2-hydroxy benzoate; Combi-blocks: all remaining aromatic aldehydes and carboxylic acids; Cambridge Isotope Laboratories: all NMR solvents.

\subsection{General Procedure for Synthesis of Substituted Aryl Imines}

Anhydrous $\mathrm{Na}_{2} \mathrm{SO}_{4}(3.0 \mathrm{~g})$ was added to a solution of aromatic aldehyde $(1.0 \mathrm{mmol})$ in anhydrous dichloromethane (DCM) $(25 \mathrm{~mL})$. A solution of 3-(triethoxysilyl)propan-1-amine (APTES) (1 eq.) was added to the solution and the mixture stirred under $\mathrm{N}_{2}$ atmosphere. The solution was then filtered using grade 1 Whatman filter paper, the reaction flask and drying agent were rinsed with DCM $(\sim 5 \mathrm{~mL})$ and the solvent removed in vacuo.

(E)-4-(((3-(triethoxysilyl)propyl)imino)methyl)benzamide (2). 4-Formylbenzamide (0.075 g, $0.503 \mathrm{mmol}$ ) and 3-(triethoxysilyl)propan-1-amine (APTES) $(0.112 \mathrm{~g}, 0.506 \mathrm{mmol})$ were reacted as described in the general procedure to give a flaky, light-yellow solid $(0.154 \mathrm{~g}$, $0.437 \mathrm{mmol}, 87 \%) .{ }^{1} \mathrm{H}-\mathrm{NMR}\left(400 \mathrm{MHz}^{\mathrm{C}} \mathrm{CDCl}_{3}\right) \delta 8.24(\mathrm{~s}, 1 \mathrm{H}), 7.79(\mathrm{~d}, J=8.6 \mathrm{~Hz} 1 \mathrm{H}), 7.74$ $(\mathrm{d}, J=8.6 \mathrm{~Hz} 1 \mathrm{H}), 6.01(\mathrm{~s}, 1 \mathrm{H}), 5.55(\mathrm{~s}, 1 \mathrm{H}), 3.76(\mathrm{q}, J=7.0 \mathrm{~Hz}, 6 \mathrm{H}), 3.58(\mathrm{td}, J=6.9,1.4 \mathrm{~Hz}$, 2H), 1.82-1.73 (m, 2H), $1.16(\mathrm{t}, J=7.0 \mathrm{~Hz}, 9 \mathrm{H}), 0.61(\mathrm{~m}, 2 \mathrm{H}) .{ }^{13} \mathrm{C}-\mathrm{NMR}\left(101 \mathrm{MHz}, \mathrm{CDCl}_{3}\right) \delta$ $168.6,159.9,139.5,134.7,128.2,127.6,64.3,58.4,24.2,18.3,8.0$. HRMS (APCI-ion trap) $m / z$ : $[\mathrm{M}+\mathrm{H}]+$ Calc for $\mathrm{C}_{17} \mathrm{H}_{28} \mathrm{~N}_{2} \mathrm{O}_{4} \mathrm{SiH}$ : 353.1897; Found: 353.1901 .

Methyl (E)-4-(((3-(triethoxysilyl)propyl)imino)methyl)benzoate (3). Methyl 4-formyl benzoate (0.075 g, $0.457 \mathrm{mmol})$ and 3-(triethoxysilyl)propan-1-amine (APTES) (0.103 g, $0.461 \mathrm{mmol}$ ) were reacted as described in the general procedure to give a flaky, lightyellow solid (0.137 g, $0.373 \mathrm{mmol}, 82 \%) .{ }^{1} \mathrm{H}-\mathrm{NMR}\left(400 \mathrm{MHz}, \mathrm{CDCl}_{3}\right) \delta 8.25(\mathrm{~s}, 1 \mathrm{H}), 8.00(\mathrm{~m}$, $2 \mathrm{H}), 7.72(\mathrm{~m}, 2 \mathrm{H}), 3.86(\mathrm{~s}, 3 \mathrm{H}), 3.76(\mathrm{q}, J=7.0 \mathrm{~Hz}, 6 \mathrm{H}), 3.58(\mathrm{td}, J=6.9,1.3 \mathrm{~Hz}, 2 \mathrm{H}), 1.83-1.71$ $(\mathrm{m}, 2 \mathrm{H}), 1.16(\mathrm{t}, J=7.0 \mathrm{~Hz}, 9 \mathrm{H}), 0.66-0.57(\mathrm{~m}, 2 \mathrm{H}) .{ }^{13} \mathrm{C}-\mathrm{NMR}\left(101 \mathrm{MHz}, \mathrm{CDCl}_{3}\right) \delta 165.7$, 159.0, 139.2, 130.6, 128.8, 126.8, 63.4, 57.3, 51.2, 23.1, 17.2, 7.0. HRMS (APCI-ion trap) $m / z$ : $[\mathrm{M}+\mathrm{H}]+$ Calc for $\mathrm{C}_{18} \mathrm{H}_{29} \mathrm{NO}_{5} \mathrm{SiH}: 368.1893$; Found: 368.1889 .

(E)-N-methyl-4-(((3-(triethoxysilyl)propyl)imino)methyl)benzamide (4). 4-Formyl-N-methyl benzamide $(0.050 \mathrm{~g}, 0.306 \mathrm{mmol}$ ) and 3-(triethoxysilyl)propan-1-amine (APTES) $(0.068 \mathrm{~g}$, $0.307 \mathrm{mmol})$ were reacted as described in the general procedure to give a viscous, lightyellow liquid $(0.076 \mathrm{~g}, 0.207 \mathrm{mmol}, 68 \%)$. ${ }^{1} \mathrm{H}-\mathrm{NMR}\left(400 \mathrm{MHz}, \mathrm{CDCl}_{3}\right) \delta 8.23(\mathrm{~s}, 1 \mathrm{H})$, 7.75-7.68 (m, 4H), $6.11(\mathrm{~s}, 1 \mathrm{H}), 3.76(\mathrm{q}, J=7.0 \mathrm{~Hz}, 6 \mathrm{H}), 3.57(\mathrm{td}, J=7.0,1.3 \mathrm{~Hz}, 2 \mathrm{H}), 2.96(\mathrm{~d}$, $J=4.9 \mathrm{~Hz}, 3 \mathrm{H}), 1.77(\mathrm{~m}, 2 \mathrm{H}), 1.16(\mathrm{t}, J=7.0 \mathrm{~Hz}, 9 \mathrm{H}), 0.67-0.56(\mathrm{~m}, 2 \mathrm{H}) .{ }^{13} \mathrm{C}-\mathrm{NMR}(101 \mathrm{MHz}$, $\left.\mathrm{CDCl}_{3}\right) \delta 167.6,160.0,138.9,136.1,128.1,127.1,64.3,58.4,26.9,24.2,18.3,8.0$. HRMS (APCI-ion trap) $m / z$ : $[\mathrm{M}+\mathrm{H}]+$ Calc for $\mathrm{C}_{18} \mathrm{H}_{30} \mathrm{~N}_{2} \mathrm{O}_{4} \mathrm{SiH}: 367.2053$; Found: 367.2052. 
(E)-1-(4-((3-(triethoxysilyl)propyl)imino)methyl)phenyl)ethan-1-one (5). 4-Acetyl benzaldehyde (0.074 g, $0.499 \mathrm{mmol})$ and 3-(triethoxysilyl)propan-1-amine (APTES) (0.112 g, $0.506 \mathrm{mmol})$ were reacted as described in the general procedure to give a viscous, light-yellow liquid (0.142 g, $0.404 \mathrm{mmol}, 81 \%) .{ }^{1} \mathrm{H}-\mathrm{NMR}\left(400 \mathrm{MHz}, \mathrm{CDCl}_{3}\right) \delta 8.25$ (s, 1H), 7.91 (d, J = 8.4 Hz, $2 \mathrm{H}), 7.74(\mathrm{~d}, J=8.4 \mathrm{~Hz}, 2 \mathrm{H}), 3.76(\mathrm{q}, J=7.0 \mathrm{~Hz}, 6 \mathrm{H}), 3.58(\mathrm{~m}, 2 \mathrm{H}), 2.55(\mathrm{~s}, 3 \mathrm{H}), 1.85-1.71$ $(\mathrm{m}, 2 \mathrm{H}), 1.16(\mathrm{t}, J=7.0 \mathrm{~Hz}, 9 \mathrm{H}), 0.68-0.54(\mathrm{~m}, 2 \mathrm{H}) .{ }^{13} \mathrm{C}-\mathrm{NMR}\left(101 \mathrm{MHz}, \mathrm{CDCl}_{3}\right) \delta 197.7$, $159.9,140.2,138.3,128.5,128.1,64.4,58.3,26.7,24.1,18.3,8.0$. HRMS (APCI-ion trap) $m / z$ : $[\mathrm{M}+\mathrm{H}]+$ Calc for $\mathrm{C}_{18} \mathrm{H}_{29} \mathrm{NO}_{4} \mathrm{SiH}: 352.1944$; Found: 352.1945 .

(1E,1'E)-1,1 -(1,4-phenylene)bis(N-(3-(triethoxysilyl)propyl)methanimine) (6). Teraphthalaldehyde (0.134 g, $1 \mathrm{mmol}$ ) and 3-(triethoxysilyl)propan-1-amine (APTES) (0.442 g, $2 \mathrm{mmol})$ were reacted as described in the general procedure to give a viscous light-yellow liquid (0.282 g, $0.521 \mathrm{mmol}, 52 \%) .{ }^{1} \mathrm{H}-\mathrm{NMR}\left(400 \mathrm{MHz} \mathrm{CDCl}_{3}\right) \delta 8.22(\mathrm{~s}, 2 \mathrm{H}), 7.69(\mathrm{~s}, 4 \mathrm{H}), 3.75$ $(\mathrm{q}, J=7.0 \mathrm{~Hz}, 12 \mathrm{H}), 3.56(\mathrm{td}, J=6.9,1.3 \mathrm{~Hz}, 4 \mathrm{H}), 1.82-1.72(\mathrm{~m}, 4 \mathrm{H}), 1.16(\mathrm{t}, J=7.0 \mathrm{~Hz}$, 18H), 0.65-0.58 (m, 4H). ${ }^{13} \mathrm{C}-\mathrm{NMR}\left(101 \mathrm{MHz}, \mathrm{CDCl}_{3}\right) \delta 160.5,138.1,128.2,64.4,53.3,24.2$, 18.3, 8.0. HRMS (APCI-ion trap) $m / z$ : $[\mathrm{M}+\mathrm{H}]+$ Calc for $\mathrm{C}_{26} \mathrm{H}_{48} \mathrm{~N}_{2} \mathrm{O}_{6} \mathrm{SiH}: 541.3129$; Found: 541.3126.

(E)-2-(((3-(triethoxysilyl)propyl)imino)methyl)benzonitrile. (8). 2-Cyanobenzaldehyde (0.131 g, $1.0 \mathrm{mmol}$ ) and 3-(triethoxysilyl)propan-1-amine (APTES) $(0.221 \mathrm{~g}, 1.0 \mathrm{mmol})$ were reacted as described in the general procedure to give a viscous light red liquid $(0.270 \mathrm{~g}, 0.80 \mathrm{mmol}$, 80\%). ${ }^{1} \mathrm{H}-\mathrm{NMR}\left(400 \mathrm{MHz}, \mathrm{CDCl}_{3}\right) \delta 8.66(\mathrm{~s}, 1 \mathrm{H}), 8.13(\mathrm{~d}, J=8.0 \mathrm{~Hz}, 1 \mathrm{H}), 7.70(\mathrm{~d}, J=8.0 \mathrm{~Hz}$, $1 \mathrm{H}), 7.63(\mathrm{t}, J=8.0 \mathrm{~Hz}, 1 \mathrm{H}), 7.51(\mathrm{t}, J=8.0 \mathrm{~Hz}, 1 \mathrm{H}), 3.83(\mathrm{q}, J=8.0 \mathrm{~Hz}, 6 \mathrm{H}), 3.73-3.70(\mathrm{~m}$, $2 \mathrm{H}), 1.90-1.82(\mathrm{~m}, 2 \mathrm{H}), 1.24(\mathrm{t}, J=8.0 \mathrm{~Hz}, 9 \mathrm{H}), 0.72-0.68(\mathrm{~m}, 2 \mathrm{H}) .{ }^{13} \mathrm{C}-\mathrm{NMR}(101 \mathrm{MHz}$, $\left.\mathrm{CDCl}_{3}\right) \delta 156.9,138.5,132.9,132.8,130.4,127.3,117.0,112.6,64.3,58.4,24.2,18.3$, 8.0. HRMS (APCI-ion trap) $m / z:[\mathrm{M}+\mathrm{H}]+$ Calc for $\mathrm{C}_{17} \mathrm{H}_{26} \mathrm{~N}_{2} \mathrm{O}_{3} \mathrm{SiH}$ : 335.1791; Found: 335.1789.

(E)-3-(((3-(triethoxysilyl)propyl)imino)methyl)benzonitrile (9). 3-Cyanobenzaldehyde (0.131 g, $1.0 \mathrm{mmol}$ ) and 3-(triethoxysilyl)propan-1-amine (APTES) $(0.221 \mathrm{~g}, 1.0 \mathrm{mmol}$ ) were reacted as described in the general procedure to give a viscous pale yellow liquid $(0.270 \mathrm{~g}, 0.80 \mathrm{mmol}$, $80.0 \%) .{ }^{1} \mathrm{H}-\mathrm{NMR}\left(400 \mathrm{MHz}, \mathrm{CDCl}_{3}\right) \delta 8.27(\mathrm{~s}, 1 \mathrm{H}), 8.03(\mathrm{~s}, 1 \mathrm{H}), 7.94(\mathrm{~d}, J=8.0 \mathrm{~Hz}, 1 \mathrm{H})$, $7.69(\mathrm{~d}, J=8.0 \mathrm{~Hz}, 1 \mathrm{H}), 7.52(\mathrm{t}, J=8.0 \mathrm{~Hz}, 1 \mathrm{H}), 3.83(\mathrm{q}, J=8.0 \mathrm{~Hz}, 6 \mathrm{H}), 3.66-3.63(\mathrm{~m}, 2 \mathrm{H})$, $1.88-1.80(\mathrm{~m}, 2 \mathrm{H}), 1.23(\mathrm{t}, J=8.0 \mathrm{~Hz}, 9 \mathrm{H}), 0.70-0.65(\mathrm{~m}, 2 \mathrm{H}) .{ }^{13} \mathrm{C}-\mathrm{NMR}\left(101 \mathrm{MHz}, \mathrm{CDCl}_{3}\right) \delta$ $158.4,137.4,133.5,132.0,131.5,129.4,118.3,112.9,64.1,58.4,24.1,18.3,8.0$. HRMS (APCI-ion trap) $m / z:[\mathrm{M}+\mathrm{H}]+$ Calc for $\mathrm{C}_{17} \mathrm{H}_{26} \mathrm{~N}_{2} \mathrm{O}_{3} \mathrm{SiH}$ : 335.1791; Found: 335.1800.

(E)-2-fluoro-4-(((3-(triethoxysilyl)propyl)imino)methyl)benzonitrile (10). 2-Fluoro-4-formyl benzonitrile $(0.075 \mathrm{~g}, 0.503 \mathrm{mmol})$ and 3-(triethoxysilyl)propan-1-amine (APTES) (0.112 g, $0.506 \mathrm{mmol})$ were reacted as described in the general procedure to give a viscous, lightyellow liquid (0.120 g, $0.340 \mathrm{mmol}, 68 \%)$. ${ }^{1} \mathrm{H}-\mathrm{NMR}\left(400 \mathrm{MHz}, \mathrm{CDCl}_{3}\right) \delta 8.19(\mathrm{~s}, 1 \mathrm{H}), 7.58$ $(\mathrm{dd}, J=8.0,4.0 \mathrm{~Hz}, 1 \mathrm{H}), 7.55(\mathrm{dd}, J=9.6,1.4 \mathrm{~Hz}, 1 \mathrm{H}), 7.50(\mathrm{dd}, J=8.0,1.4 \mathrm{~Hz}, 1 \mathrm{H}), 3.76(\mathrm{q}$, $J=7.0 \mathrm{~Hz}, 6 \mathrm{H}), 3.59(\mathrm{td}, J=6.9,1.4 \mathrm{~Hz}, 2 \mathrm{H}), 1.82-1.71(\mathrm{~m}, 2 \mathrm{H}), 1.16(\mathrm{t}, J=7.0 \mathrm{~Hz}, 9 \mathrm{H}), 0.65-$ $0.54(\mathrm{~m}, 2 \mathrm{H}) .{ }^{13} \mathrm{C}-\mathrm{NMR}\left(101 \mathrm{MHz}, \mathrm{CDCl}_{3}\right) \delta 163.3(\mathrm{~d}, J=259.6 \mathrm{~Hz}) 157.8(\mathrm{~d}, J=2.8 \mathrm{~Hz}), 143.0$ $(\mathrm{d}, J=7.5 \mathrm{~Hz}), 133.7,124.3(\mathrm{~d}, J=3.4 \mathrm{~Hz}), 115.0(\mathrm{~d}, J=20.6 \mathrm{~Hz}), 113.7,102.7(\mathrm{~d}, J=16.1 \mathrm{~Hz})$, 64.1, 58.4, 24.1, 18.3, 8.1. HRMS (APCI-ion trap) $m / z$ : $[\mathrm{M}+\mathrm{H}]+$ Calc for $\mathrm{C}_{17} \mathrm{H}_{25} \mathrm{~N}_{2} \mathrm{O}_{3} \mathrm{FSiH}$ : 353.1697; Found: 361.1688 .

(E)-1-(4-nitrophenyl)-N-(3-(triethoxysilyl)propyl)methanimine (11). 4-Nitrobenzaldehyde $(0.151 \mathrm{~g}, 1.0 \mathrm{mmol})$ and 3-(triethoxysilyl)propan-1-amine (APTES) (0.221 g, $1.0 \mathrm{mmol})$ were reacted as described in the general procedure to give a viscous lightly tinged liquid $(0.305 \mathrm{~g}, 0.86 \mathrm{mmol}, 86 \%){ }^{1} \mathrm{H}-\mathrm{NMR}\left(400 \mathrm{MHz}, \mathrm{CDCl}_{3}\right) \delta 8.37(\mathrm{~s}, 1 \mathrm{H}), 8.28$ (d, J = 8.0 Hz, $2 \mathrm{H}), 7.91(\mathrm{~d}, J=8.0 \mathrm{~Hz}, 2 \mathrm{H}), 3.86(\mathrm{q}, J=8.0 \mathrm{~Hz}, 6 \mathrm{H}), 3.72-3.68(\mathrm{~m}, 2 \mathrm{H}), 1.91-1.84(\mathrm{~m}, 2 \mathrm{H})$, $1.25(\mathrm{t}, J=8.0 \mathrm{~Hz}, 9 \mathrm{H}), 0.72-0.68(\mathrm{~m}, 2 \mathrm{H}) .{ }^{13} \mathrm{C}-\mathrm{NMR}\left(101 \mathrm{MHz}, \mathrm{CDCl}_{3}\right) \delta 154.6,148.9,141.8$, $128.7,123.8,64.4,58.4,24.1,18.3,8.1$. HRMS (APCI-ion trap) $m / z$ : $[\mathrm{M}+\mathrm{H}]+$ Calc for $\mathrm{C}_{16} \mathrm{H}_{26} \mathrm{~N}_{2} \mathrm{O}_{5} \mathrm{SiH}: 355.1689$; Found: 355.1687.

(E)-1-(pyridin-4-yl)-N-(3-(triethoxysilyl)propyl)methanimine (13). Isonicotinaldehyde (0.107 $\mathrm{g}$, $1.0 \mathrm{mmol}$ ) and 3-(triethoxysilyl)propan-1-amine (APTES) $(0.221 \mathrm{~g}, 1.0 \mathrm{mmol})$ were reacted 
as described in the general procedure to give a clear liquid $(0.185 \mathrm{~g}, 0.6 \mathrm{mmol}, 60 \%) .{ }^{1} \mathrm{H}-$ $\operatorname{NMR}\left(400 \mathrm{MHz}, \mathrm{CDCl}_{3}\right) \delta 8.68(\mathrm{~d}, J=8.0 \mathrm{~Hz}, 2 \mathrm{H}), 8.26(\mathrm{~s}, 1 \mathrm{H}), 7.58(\mathrm{~d}, J=8.0 \mathrm{~Hz}, 2 \mathrm{H}), 3.83$ $(\mathrm{q}, J=8.0 \mathrm{~Hz}, 6 \mathrm{H}), 3.68-3.65(\mathrm{~m}, 2 \mathrm{H}), 1.88-1.81(\mathrm{~m}, 2 \mathrm{H}), 1.23(\mathrm{t}, J=8.0 \mathrm{~Hz}, 9 \mathrm{H}), 0.70-0.65$ $(\mathrm{m}, 2 \mathrm{H}) .{ }^{13} \mathrm{C}-\mathrm{NMR}\left(101 \mathrm{MHz}, \mathrm{CDCl}_{3}\right) \delta 159.0,150.4,143.0,121.9,64.3,58.4,24.0,18.3$, 8.0. HRMS (APCI-ion trap) $m / z:[\mathrm{M}+\mathrm{H}]+$ Calc for $\mathrm{C}_{15} \mathrm{H}_{26} \mathrm{~N}_{2} \mathrm{O}_{3} \mathrm{SiH}: 311.1790$; Found: 311.1788.

(E)-1-(pyridazin-4-yl)-N-(3-(triethoxysilyl)propyl)methanimine (14). Pyridazine-4- carbaldehyde $(0.050 \mathrm{~g}, 0.463 \mathrm{mmol})$ and 3-(triethoxysilyl)propan-1-amine (APTES) (0.103 g, $0.465 \mathrm{mmol}$ ) were reacted as described in the general procedure to give a viscous, lightyellow liquid (0.094 g, $0.302 \mathrm{mmol}, 65 \%) .{ }^{1} \mathrm{H}-\mathrm{NMR}\left(400 \mathrm{MHz}, \mathrm{CDCl}_{3}\right) \delta 9.41(\mathrm{dd}, J=2.2$, $1.3 \mathrm{~Hz}, 1 \mathrm{H}), 9.21(\mathrm{dd}, J=5.2,1.3 \mathrm{~Hz}, 1 \mathrm{H}), 8.22(\mathrm{~s}, 1 \mathrm{H}), 7.65(\mathrm{dd}, J=5.2,2.2 \mathrm{~Hz}, 1 \mathrm{H}), 3.76$ $(\mathrm{q}, J=7.0 \mathrm{~Hz}, 6 \mathrm{H}), 3.64(\mathrm{td}, J=6.9,1.5 \mathrm{~Hz}, 2 \mathrm{H}), 1.89-1.71(\mathrm{~m}, 2 \mathrm{H}), 1.16(\mathrm{t}, J=7.0 \mathrm{~Hz}, 9 \mathrm{H})$, 0.71-0.51 (m, 2H). ${ }^{13} \mathrm{C}-\mathrm{NMR}\left(101 \mathrm{MHz}, \mathrm{CDCl}_{3}\right) \delta 156.3,151.8,149.8,133.2,123.7,64.5,58.4$, 24.0, 18.3, 8.1. HRMS (APCI-ion trap) $m / z$ : [M + H] + Calc for $\mathrm{C}_{14} \mathrm{H}_{25} \mathrm{~N}_{3} \mathrm{O}_{3} \mathrm{SiH}$ : 312.1743; Found: 312.1742 .

(E)-1-(4-(pyridin-4-yl)phenyl)-N-(3-(triethoxysilyl)propyl)methanimine (15). 4-(Pyridin-4-yl) benzaldehyde $(0.183 \mathrm{~g}, 1.0 \mathrm{mmol})$ and 3-(triethoxysilyl)propan-1-amine (APTES) $(0.221 \mathrm{~g}$, $1.0 \mathrm{mmol}$ ) were reacted as described in the general procedure to give a viscous liquid $(0.325 \mathrm{~g}, 0.84 \mathrm{mmol}, 84 \%){ }^{1} \mathrm{H}-\mathrm{NMR}\left(400 \mathrm{MHz}, \mathrm{CDCl}_{3}\right) \delta 8.68(\mathrm{~d}, J=8.0 \mathrm{~Hz}, 2 \mathrm{H}), 8.33(\mathrm{~s}$, $1 \mathrm{H}), 7.84(\mathrm{~d}, J=8.0 \mathrm{~Hz}, 2 \mathrm{H}), 7.69(\mathrm{~d}, J=8.0 \mathrm{~Hz}, 2 \mathrm{H}), 7.55(\mathrm{~d}, J=8.0 \mathrm{~Hz}, 2 \mathrm{H}), 3.83(\mathrm{q}$, $J=8.0 \mathrm{~Hz}, 6 \mathrm{H}), 3.67-3.64(\mathrm{~m}, 2 \mathrm{H}), 1.89-1.82(\mathrm{~m}, 2 \mathrm{H}), 1.24(\mathrm{t}, J=8.0 \mathrm{~Hz}, 9 \mathrm{H}), 0.71-0.67$ $(\mathrm{m}, 2 \mathrm{H}) .{ }^{13} \mathrm{C}-\mathrm{NMR}\left(101 \mathrm{MHz}, \mathrm{CDCl}_{3}\right) \delta 160.2,150.3,147.6,139.9,136.9,128.7,127.2,121.5$, 64.4, 58.4, 24.2, 18.3, 8.0. HRMS (APCI-ion trap) $m / z:[\mathrm{M}+\mathrm{H}]+$ Calc for $\mathrm{C}_{21} \mathrm{H}_{30} \mathrm{~N}_{2} \mathrm{O}_{3} \mathrm{SiH}$ : 387.2104; Found: 387.2107.

(E)-1-(4-(1H-imidazol-1-yl)phenyl)-N-(3-(triethoxysilyl)propyl)methan-1-imine (16). 4-(1Himidazol-1-yl) benzaldehyde $(0.075 \mathrm{~g}, 0.436 \mathrm{mmol})$ and 3-(triethoxysilyl)propan-1-amine (APTES) $(0.097 \mathrm{~g}, 0.438 \mathrm{mmol})$ were reacted as described in the general procedure to give a viscous, light-yellow liquid ( $0.120 \mathrm{~g}, 0.452 \mathrm{mmol}, 73 \%) .{ }^{1} \mathrm{H}-\mathrm{NMR}\left(400 \mathrm{MHz}, \mathrm{CDCl}_{3}\right)$ $\delta 7.94(\mathrm{~s}, 1 \mathrm{H}), 7.57-7.54(\mathrm{~m}, 3 \mathrm{H}), 7.27(\mathrm{~s}, 1 \mathrm{H}), 6.73-6.67(\mathrm{~m}, 2 \mathrm{H}), 3.82(\mathrm{q}, J=7.0 \mathrm{~Hz}, 6 \mathrm{H})$, $3.59(\mathrm{td}, J=6.9,1.4 \mathrm{~Hz}, 2 \mathrm{H}), 2.13-2.03(\mathrm{~m}, 2 \mathrm{H}), 1.18(\mathrm{t}, J=7.0 \mathrm{~Hz}, 9 \mathrm{H}), 0.89-0.82(\mathrm{~m}, 2 \mathrm{H})$. ${ }^{13} \mathrm{C}-\mathrm{NMR}\left(101 \mathrm{MHz}, \mathrm{CDCl}_{3}\right) \delta$ 158.6, 138.5, 135.2, 130.9, 129.2, 120.5, 117.2, 64.2, 58.2, 24.7, 18.2, 8.5. HRMS (APCI-ion trap) $m / z:[\mathrm{M}+\mathrm{H}]+$ Calc for $\mathrm{C}_{19} \mathrm{H}_{29} \mathrm{~N}_{3} \mathrm{O}_{3} \mathrm{SiH}$ : 376.2056; Found: 376.2042 .

(E)-1-(4-(1H-pyrrol-1-yl)phenyl)-N-(3-(triethoxysilyl)propyl)methan-1-imine (17). 4-(1H-pyrazol1-yl) benzaldehyde $(0.075 \mathrm{~g}, 0.436 \mathrm{mmol}$ ) and 3-(triethoxysilyl)propan-1-amine (APTES) $(0.097 \mathrm{~g}, 0.438 \mathrm{mmol})$ were reacted as described in the general procedure to give a viscous, light-yellow liquid $(0.131 \mathrm{~g}, 0.452 \mathrm{mmol}, 80 \%) .{ }^{1} \mathrm{H}-\mathrm{NMR}\left(400 \mathrm{MHz}, \mathrm{CDCl}_{3}\right) \delta 8.21(\mathrm{~s}, 1 \mathrm{H})$, $7.90(\mathrm{dd}, J=2.5,0.6 \mathrm{~Hz}, 1 \mathrm{H}), 7.79-7.72(\mathrm{~m}, 2 \mathrm{H}), 7.72-7.65(\mathrm{~m}, 3 \mathrm{H}), 6.42(\mathrm{dd}, J=2.5,1.8 \mathrm{~Hz}$, $1 \mathrm{H}), 3.76(\mathrm{q}, J=7.0 \mathrm{~Hz}, 6 \mathrm{H}), 3.56(\mathrm{td}, J=6.9,1.3 \mathrm{~Hz}, 2 \mathrm{H}), 1.83-1.73(\mathrm{~m}, 2 \mathrm{H}), 1.16(\mathrm{t}, J=7.0 \mathrm{~Hz}$, 9H), 0.68-0.58 (m, 2H). ${ }^{13} \mathrm{C}-\mathrm{NMR}\left(101 \mathrm{MHz}, \mathrm{CDCl}_{3}\right) \delta 159.9,141.51,141.50,134.4,129.2$, 126.7, 118.9, 108.0, 64.3, 58.4, 24.2, 18.3, 8.0. HRMS (APCI-ion trap) $m / z:[\mathrm{M}+\mathrm{H}]+$ Calc for $\mathrm{C}_{19} \mathrm{H}_{29} \mathrm{~N}_{3} \mathrm{O}_{3} \mathrm{SiH}: 376.2056$; Found: 376.2046 .

(E)-1-(isoquinolin-6-yl)-N-(3-(triethoxysilyl)propyl)methanimine (18). Isoquinoline-6- carbaldehyde $(0.075 \mathrm{~g}, 0.477 \mathrm{mmol})$ and 3-(triethoxysilyl)propan-1-amine (APTES) (0.106 $\mathrm{g}$, $0.479 \mathrm{mmol}$ ) were reacted as described in the general procedure to give a viscous, lightyellow liquid (0.104 g, $0.288 \mathrm{mmol}, 60 \%) .{ }^{1} \mathrm{H}-\mathrm{NMR}\left(400 \mathrm{MHz}, \mathrm{CDCl}_{3}\right) \delta 9.20(\mathrm{~s}, 1 \mathrm{H}), 8.50$ $(\mathrm{d}, J=5.7 \mathrm{~Hz}, 1 \mathrm{H}), 8.39(\mathrm{~d}, J=1.5 \mathrm{~Hz}, 1 \mathrm{H}), 8.04(\mathrm{dd}, J=8.5,1.6 \mathrm{~Hz}, 1 \mathrm{H}), 7.98-7.93(\mathrm{~m}, 2 \mathrm{H})$, $7.63(\mathrm{dt}, J=5.8,1.0 \mathrm{~Hz}, 1 \mathrm{H}), 3.77(\mathrm{q}, J=7.0 \mathrm{~Hz}, 6 \mathrm{H}), 3.63(\mathrm{td}, J=6.9,1.4 \mathrm{~Hz}, 2 \mathrm{H}), 1.88-1.75$ $(\mathrm{m}, 2 \mathrm{H}), 1.17(\mathrm{t}, J=7.0 \mathrm{~Hz}, 9 \mathrm{H}), 0.70-0.58(\mathrm{~m}, 2 \mathrm{H}) .{ }^{13} \mathrm{C}-\mathrm{NMR}\left(101 \mathrm{MHz}, \mathrm{CDCl}_{3}\right) \delta 160.2$, 152.4, 143.7, 138.0, 135.7, 129.4, 128.0, 127.7, 125.5, 120.8, 64.4, 58.4, 24.2, 18.3, 8.1. HRMS (APCI-ion trap) $m / z:[\mathrm{M}+\mathrm{H}]+$ Calc for $\mathrm{C}_{19} \mathrm{H}_{28} \mathrm{NO}_{3} \mathrm{SiH}$ : 361.1947; Found: 361.1937. 
(E)-1-(9H-carbazol-3-yl)-N-(3-(triethoxysilyl)propyl)methanimine (19). 9H-Carbazole-3-carbal dehyde (0.195 g, $1.0 \mathrm{mmol}$ ) and 3-(triethoxysilyl)propan-1-amine (APTES) (0.221 g, $1.0 \mathrm{mmol})$ were reacted as described in the general procedure to give a viscous liquid $(0.260 \mathrm{~g}$, $0.66 \mathrm{mmol}, 65 \%) .{ }^{1} \mathrm{H}-\mathrm{NMR}\left(400 \mathrm{MHz}, \mathrm{CDCl}_{3}\right) \delta 8.20(\mathrm{br} \mathrm{s}, 1 \mathrm{H}), 8.16(\mathrm{~s}, 1 \mathrm{H}), 8.08(\mathrm{~m}, 2 \mathrm{H})$, 7.43-7.41 (m, 3H), 7.26-7.22 (m, 2H), $3.82(\mathrm{q}, J=8.0 \mathrm{~Hz}, 6 \mathrm{H}), 3.33-3.28(\mathrm{~m}, 2 \mathrm{H}), 1.70-1.62$ $(\mathrm{m}, 2 \mathrm{H}), 1.23(\mathrm{t}, J=8.0 \mathrm{~Hz}, 9 \mathrm{H}), 0.67-0.63(\mathrm{~m}, 2 \mathrm{H}) .{ }^{13} \mathrm{C}-\mathrm{NMR}\left(101 \mathrm{MHz}, \mathrm{CDCl}_{3}\right) \delta 164.6$, 161.1, 139.5, 125.8, 123.3, 120.3, 119.3, 110.6, 58.5, 40.4, 22.8, 18.3, 7.7. HRMS (APCI-ion trap) $m / z:[\mathrm{M}+\mathrm{H}]+$ Calc for $\mathrm{C}_{22} \mathrm{H}_{30} \mathrm{~N}_{2} \mathrm{O}_{3} \mathrm{SiH}: 399.2104$; Found: 399.2105.

Methyl (E)-2-hydroxy-4-(((3-(triethoxysilyl)propyl)imino)methyl)benzoate (21). Methyl 2-hydroxy 4-formyl benzoate $(0.050 \mathrm{~g}, 0.278 \mathrm{mmol})$ and 3-(triethoxysilyl)propan-1-amine (APTES) $(0.062 \mathrm{~g}, 0.280 \mathrm{mmol})$ were reacted as described in the general procedure to give a viscous, light-yellow liquid (0.093 g, $0.242 \mathrm{mmol}, 87 \%) .{ }^{1} \mathrm{H}-\mathrm{NMR}\left(400 \mathrm{MHz}, \mathrm{CDCl}_{3}\right) \delta 8.16(\mathrm{~s}, 1 \mathrm{H})$, $7.79(\mathrm{~d}, J=8.2 \mathrm{~Hz}, 1 \mathrm{H}), 7.24(\mathrm{dd}, J=8.3,1.6 \mathrm{~Hz}, 1 \mathrm{H}), 7.19(\mathrm{~d}, J=1.5 \mathrm{~Hz}, 1 \mathrm{H}), 3.89(\mathrm{~s}, 3 \mathrm{H}), 3.76$ $(\mathrm{q}, J=7.0 \mathrm{~Hz}, 6 \mathrm{H}), 1.83-1.71(\mathrm{~m}, 2 \mathrm{H}), 1.16(\mathrm{t}, J=7.0 \mathrm{~Hz}, 9 \mathrm{H}), 0.66-0.58(\mathrm{~m}, 2 \mathrm{H}) .{ }^{13} \mathrm{C}-\mathrm{NMR}$ $\left(101 \mathrm{MHz}_{1} \mathrm{CDCl}_{3}\right) \delta 170.3,161.7,159.9,142.9,130.1,118.0,117.4,113.6,64.3,58.4,52.4,24.1$, 18.3, 8.0. HRMS (APCI-ion trap) $m / z$ : $[\mathrm{M}+\mathrm{H}]+$ Calc for $\mathrm{C}_{18} \mathrm{H}_{29} \mathrm{NO}_{6} \mathrm{SiH}$ : 384.1842; Found: 384.1846 .

Methyl (E)-2-methyl-4-(((3-(triethoxysilyl)propyl)imino)methyl)benzoate (22). Methyl 4-formyl2-methyl benzoate $(0.089 \mathrm{~g}, 0.500 \mathrm{mmol})$ and 3-(triethoxysilyl)propan-1-amine (APTES) $(0.111 \mathrm{~g}, 0.501 \mathrm{mmol})$ were reacted as described in the general procedure to give an offwhite flaky solid $(0.182 \mathrm{~g}, 0.477 \mathrm{mmol}, 56 \%){ }^{1} \mathrm{H}-\mathrm{NMR}\left(400 \mathrm{MHz}, \mathrm{CDCl}_{3}\right) \delta 8.20(\mathrm{~d}, J=1.3 \mathrm{~Hz}$, $1 \mathrm{H}), 7.87(\mathrm{~d}, J=8.0 \mathrm{~Hz}, 1 \mathrm{H}), 7.54(\mathrm{~d}, J=1.6 \mathrm{~Hz}, 1 \mathrm{H}), 7.49(\mathrm{dd}, J=8.1,1.7 \mathrm{~Hz}, 1 \mathrm{H}), 3.83(\mathrm{~s}$, $3 \mathrm{H}), 3.76(\mathrm{q}, J=7.0 \mathrm{~Hz}, 6 \mathrm{H}), 3.56(\mathrm{td}, J=6.9,1.4 \mathrm{~Hz}, 2 \mathrm{H}), 2.56(\mathrm{~s}, 3 \mathrm{H}), 1.83-1.72(\mathrm{~m}, 2 \mathrm{H})$, $1.16(\mathrm{t}, J=7.0 \mathrm{~Hz}, 9 \mathrm{H}), 0.65-0.58(\mathrm{~m}, 2 \mathrm{H}) .{ }^{13} \mathrm{C}-\mathrm{NMR}\left(101 \mathrm{MHz}, \mathrm{CDCl}_{3}\right) \delta 167.6,160.2,140.5$, $139.1,131.1,130.93,130.92,125.3,64.4,58.4,51.9,24.2,21.6,18.3,8.0$. HRMS (APCI-ion trap) $m / z:[\mathrm{M}+\mathrm{H}]+$ Calc for $\mathrm{C}_{19} \mathrm{H}_{31} \mathrm{NO}_{5} \mathrm{SiH}$ : 382.2050; Found: 382.2052.

\subsection{General Procedure for Synthesis of Substituted Aryl Amides}

Substituted aromatic benzoic acid $(1.0 \mathrm{mmol}), N$-hydroxysuccinimide (NHS) (1.5 equivalent), and $N, N$-dicyclohexylcarbodiimide (DCC) (1.2 equivalent) were dissolved in anhydrous THF $(10 \mathrm{~mL})$ in a $100 \mathrm{~mL}$ round bottom flask and stirred under nitrogen atmosphere for $4 \mathrm{~h}$ at room temperature. The precipitate was filtered and 3-(triethoxysilyl) propan-1amine (APTES) (1.0 equivalent) and triethylamine (TEA) (1.0 equivalent) were added to the clear filtrate. The solution was then stirred for $12 \mathrm{~h}$ at room temperature under $\mathrm{N}_{2}$. The precipitate was filtered, and solvent removed in vacuo. The crude material was purified via flash chromatography on silica gel using ethyl acetate as a mobile phase.

$\mathrm{N}$-(3-(triethoxysilyl)propyl)isonicotinamide (24). Isonicotinic acid (0.123 g, $1.0 \mathrm{mmol}), \mathrm{N}$ hydroxysuccinimide (NHS) ( $0.173 \mathrm{~g}, 1.5$ equivalent), and $N, N$-dicyclohexylcarbodiimide (DCC) (0.248 g, 1.2 equivalent) were reacted with APTES and TEA and the crude product chromatographed as described in the general procedure to yield a viscous liquid with a yellow tinge $(0.165 \mathrm{~g}, 0.51 \mathrm{mmol}, 51 \%)$ upon removing organic solvents in vacuo. ${ }^{1} \mathrm{H}-\mathrm{NMR}$ $\left(400 \mathrm{MHz} \mathrm{CDCl}_{3}\right) \delta 8.72(\mathrm{~d}, J=8.0 \mathrm{~Hz}, 2 \mathrm{H}), 7.63(\mathrm{~d}, J=8.0 \mathrm{~Hz}, 2 \mathrm{H}), 6.88(\mathrm{~s}, 1 \mathrm{H}), 3.81(\mathrm{q}$, $J=7.0 \mathrm{~Hz}, 6 \mathrm{H}), 3.49-3.44(\mathrm{~m}, 2 \mathrm{H}), 1.80-1.72(\mathrm{~m}, 2 \mathrm{H}), 1.20(\mathrm{t}, J=7.0 \mathrm{~Hz}, 9 \mathrm{H}), 0.742-0.68$ (m, 2H). ${ }^{13} \mathrm{C}-\mathrm{NMR}\left(101 \mathrm{MHz}, \mathrm{CDCl}_{3}\right) \delta 165.4,150.2,142.2,121.0,58.6,42.2,22.6,18.2$, 7.8. HRMS (APCI-ion trap) $m / z$ : $[\mathrm{M}+\mathrm{H}]+$ Calc for $\mathrm{C}_{15} \mathrm{H}_{26} \mathrm{~N}_{2} \mathrm{O}_{4} \mathrm{SiH}$ : 327.1740; Found: 327.1740 .

N-(3-(triethoxysilyl)propyl)benzofuran-5-carboxamide (25). Benzofuran-5-carboxylic acid (0.162 g, $1.0 \mathrm{mmol}), N$-hydroxysuccinimide (NHS) (0.173 g, 1.5 equivalent), and $N, N-$ dicyclohexy lcarbodiimide (DCC) $(0.248 \mathrm{~g}, 1.2$ equivalent) were reacted with APTES and TEA and the crude product chromatographed as described in the general procedure to yield a viscous pale yellow liquid $(0.201 \mathrm{~g}, 0.55 \mathrm{mmol}, 55 \%)$ upon removing organic solvents in vacuo. ${ }^{1} \mathrm{H}-\mathrm{NMR}\left(400 \mathrm{MHz}, \mathrm{CDCl}_{3}\right) \delta 8.07(\mathrm{~s}, 1 \mathrm{H}), 7.74(\mathrm{~d}, J=8.0 \mathrm{~Hz}, 1 \mathrm{H}), 7.68(\mathrm{~s}, 1 \mathrm{H}), 7.52$ $(\mathrm{d}, J=8.0 \mathrm{~Hz}, 1 \mathrm{H}), 6.82(\mathrm{~s}, 1 \mathrm{H}), 6.56(\mathrm{br} \mathrm{s}, 1 \mathrm{H}), 3.83(\mathrm{q}, J=8.0 \mathrm{~Hz}, 6 \mathrm{H}), 3.52-3.47(\mathrm{~m}, 2 \mathrm{H})$, 
1.82-1.72 (m, 2H), $1.23(\mathrm{t}, J=8.0 \mathrm{~Hz}, 9 \mathrm{H}), 0.75-0.71(\mathrm{~m}, 2 \mathrm{H}) .{ }^{13} \mathrm{C}-\mathrm{NMR}\left(101 \mathrm{MHz}, \mathrm{CDCl}_{3}\right) \delta$ $167.7,156.4,146.1,130.1,127.4,123.3,120.5,111.2,106.9,58.5,42.3,22.9,18.3,7.8$. HRMS (APCI-ion trap) $m / z:[\mathrm{M}+\mathrm{H}]+$ Calc for $\mathrm{C}_{18} \mathrm{H}_{27} \mathrm{NO}_{5} \mathrm{SiH}: 366.1737$; Found: 366.1736 .

N-(3-(triethoxysilyl)propyl)benzo[b]thiophene-5-carboxamide (26). Benzo-[b]-thiophene-5carboxylic acid $(0.178 \mathrm{~g}, 1.0 \mathrm{mmol}), \mathrm{N}$-hydroxysuccinimide (NHS) (0.173 g, 1.5 equivalent), and $N, N$-dicyclohexylcarbodiimide (DCC) $(0.248 \mathrm{~g}, 1.2$ equivalent) were reacted with APTES and TEA as described in the general procedure. The crude material was purified via flash chromatography on $\mathrm{SiO}_{2}$ using diethyl ether and the first band collected gave a viscous clear liquid $(0.187 \mathrm{~g}, 0.49 \mathrm{mmol}, 49 \%)$ upon removing organic solvents in vacuo. ${ }^{1} \mathrm{H}-\mathrm{NMR}\left(400 \mathrm{MHz}, \mathrm{CDCl}_{3}\right) \delta 8.27(\mathrm{~s}, 1 \mathrm{H}), 7.91(\mathrm{~m}, 1 \mathrm{H}), 7.74(\mathrm{~m}, 1 \mathrm{H}), 7.52(\mathrm{~d}, J=8.0 \mathrm{~Hz}$, $1 \mathrm{H}), 7.40(\mathrm{~d}, J=8.0 \mathrm{~Hz}, 1 \mathrm{H}), 6.61$ (br s, $1 \mathrm{H}), 3.84(\mathrm{q}, J=8.0 \mathrm{~Hz}, 6 \mathrm{H}), 3.54-3.49(\mathrm{~m}, 2 \mathrm{H})$, $1.83-1.76(\mathrm{~m}, 2 \mathrm{H}), 1.23(\mathrm{t}, J=8.0 \mathrm{~Hz}, 9 \mathrm{H}), 0.76-0.72(\mathrm{~m}, 2 \mathrm{H}) .{ }^{13} \mathrm{C}-\mathrm{NMR}\left(101 \mathrm{MHz}, \mathrm{CDCl}_{3}\right) \delta$ $167.6,142.4,139.4,131.3,127.7,124.2,122.5,122.5,58.5,42.3,22.9,15.2,7.8$.

\section{Conclusions}

We successfully prepared 18 new aminopropyltriethoxysilyl-containing imines and amides using simple chemistry. We found that APTES reacted best with aromatic aldehydes when the aromatic moiety was electron deficient rather than electron rich. We also found that we could not form imines from APTES with acetophenones at room temperature or upon heating with drying agents. We hope scientists working with silicon oxide and other metal oxide surfaces will incorporate them into their surface science with the anticipation that these aromatic substituted silanes will have interesting electronic properties.

Supplementary Materials: The following data are available online, $\mathrm{MS},{ }^{1} \mathrm{H}$ and ${ }^{13} \mathrm{C}-\mathrm{NMR}$ spectra for compounds 2-6, 8-11, 13-19, 21, 22, and 24-26.

Author Contributions: S.R.B. and J.T.M. prepared all new compounds; S.R.B., J.T.M. and M.E.W. analyzed spectral data and wrote the manuscript. All authors have read and agreed to the published version of the manuscript.

Funding: This research received no external funding.

Institutional Review Board Statement: Not applicable.

Informed Consent Statement: Not applicable.

Data Availability Statement: The data presented in this study are available upon request from the corresponding author.

Acknowledgments: The authors thank Wake Forest University Center for Functional Materials for internal pilot funding of this work.

Conflicts of Interest: The authors declare no conflict of interest.

\section{References}

1. Welker, M.E. Synthesis of sulfur and silicon SAM precursors for molecular electronics applications. Phosphorus Sulfur Silicon Relat. Elem. 2020, 195, 75-87. [CrossRef]

2. Lamport, Z.A.; Broadnax, A.D.; Scharmann, B.; Bradford, R.W., III; DelaCourt, A.; Meyer, N.; Li, H.; geyer, S.M.; Thonhauser, T.; Welker, M.E.; et al. Molecular Rectifiers on Silicon: High Performance by Enhancing Top-Electrode/Molecule Coupling. ACS Appl. Mat. Interfaces 2019, 11, 18564-18570. [CrossRef] [PubMed]

3. Broadnax, A.D.; Lamport, Z.A.; Scharmann, B.; Jurchescu, O.D.; Welker, M.E. Ferrocenealkylsilane molecular rectifiers. J. Organometal. Chem. 2018, 856, 23-26. [CrossRef]

4. Lamport, Z.A.; Broadnax, A.D.; Harrison, D.; Barth, K.J.; Mendenhall, L.; Hamilton, C.T.; guthold, M.; Thonhauser, T.; Welker, M.E.; Jurchescu, O.D. Fluorinated benzalkylsilane molecular rectifiers. Sci. Rept. 2016, 6, 38092. [CrossRef] [PubMed]

5. Operamolla, A.; Punzi, A.; Farinola, g.M. Synthetic Routes to Thiol-Functionalized Organic Semiconductors for Molecular and Organic Electronics. Asian J. Org. Chem. 2017, 6, 120-138. [CrossRef]

6. Aitken, R.A.; Jethwa, S.J. Synthesis of Electro-active Compounds Suitable for Adsorption on Metal Surfaces. Org. Prep. Proced. Int. 2017, 49, 389-414. [CrossRef]

7. Ko, S.; Han, g.; Lee, J.K. Surface organic chemistry for application to organic electronics. Tetrahedron Lett. 2015, 56, 3721-3731. [CrossRef] 
8. Mallakpour, S.; Madani, M. A review of current coupling agents for modification of metal oxide nanoparticles. Prog. Org. Coat. 2015, 86, 194-207. [CrossRef]

9. Tanaka, M.; Niwa, O. Fabrication of Biosensing Interface with Monolayers. Anal. Sci. 2021, 37, 673-682. [CrossRef] [PubMed] 
10. Sheng, J.C.-C.; De La Franier, B.; Thompson, M. Assembling Surface Linker Chemistry with Minimization of Non-Specific Adsorption on Biosensor Materials. Materials 2021, 14, 472. [CrossRef] [PubMed]

11. Luo, T.; Zeng, W.-W.; Zhang, R.; Zhou, C.; Yang, X.; Ren, Z. Hydrophobic Modification of Silica Surfaces via grafting Alkoxy groups. ACS Appl. Elec. Mat. 2021, 3, 1691-1698. [CrossRef] 